\title{
Clinical prognostic factors in surgically treated oligometastatic non-small cell lung cancer: a systematic review
}

\author{
Luca Bertolaccini $^{1 \# \wedge}$, Monica Casiraghi ${ }^{1 \#}$, Giulia Sedda $^{1}$, Filippo de Marinis ${ }^{2}$, Lorenzo Spaggiari $^{1,3 \wedge}$ \\ ${ }^{1}$ Department of Thoracic Surgery, IEO, European Institute of Oncology IRCCS, Milan, Italy; ${ }^{2}$ Department of Thoracic Oncology, IEO, European \\ Institute of Oncology IRCCS, Milan, Italy; ${ }^{3}$ Department of Oncology and Hemato-Oncology, University of Milan, Milan, Italy \\ Contributions: (I) Conception and design: L Bertolaccini, M Casiraghi; (II) Administrative support: L Spaggiari; (III) Provision of study materials or \\ patients: L Bertolaccini, M Casiraghi, G Sedda; (IV) Collection and assembly of data: G Sedda; (V) Data analysis and interpretation: L Bertolaccini, \\ M Casiraghi; (VI) Manuscript writing: All authors; (VII) Final approval of manuscript: All authors. \\ "These authors equally contributed to this work. \\ Correspondence to: Dr. Luca Bertolaccini. Division of Thoracic Surgery, IEO, European Institute of Oncology IRCCS, Via Ripamonti, 435-20141 \\ Milan, Italy. Email: luca.bertolaccini@gmail.com.
}

Background: Since the concept of oligometastatic (OM) disease was introduced in the oncological scenario of non-small cell lung cancer (NSCLC), these patients progressively became a new category of stage IV NSCLC in whom the multimodality approach, including surgery, may improve prognosis. This systematic review aimed to investigate the clinical prognostic factors in OM-NSCLC surgically treated with radical intent.

Methods: This systematic review is reported following the Preferred Reporting Items for Systematic Reviews and Meta-Analyses (PRISMA) statement. Cochrane's Collaboration Tool was used to determine the risk of bias for the included studies' primary outcome. A search strategy using a combination of freetext words, relevant MeSH headings and appropriate restrictions (time limit: from January 1997 to March 2020, language: English) was designed. Potentially qualified papers were subjected to an in-depth fulltext examination after preliminary title/abstract screening to identify studies for inclusion in the systematic review. Data extracted included: study characteristics, baseline patient characteristics, primary and secondary outcomes. The Cochrane's Collaboration Tool was used to determine the risk of bias for included studies' primary outcome. The risk of bias due to incomplete outcome data was evaluated at an outcome level. However, at the study stage, the possibility of bias due to sequence generation, allocation concealment, blinding, selective reporting, or funding was assessed. Two independent observers calculated the probability of bias, and differences were resolved through dialogue and consensus.

Results: Nine studies were selected. Overall survival (OS) was 51.8 months and varied from 21.1 to 60 months, but results were not statistically significant. Positive prognostic factors for survival were cessation of smoking, age <60, a histologic grade of G1/G2, pN0. The presence of extra-brain OM and multiple metastases negatively affected survival.

Discussion: For otherwise stable patients with a single organ site with synchronous (or metachronous) extrathoracic M1 disease and no intrathoracic lymph node involvement, aggressive treatment should be used in the absence of randomized evidence to help determine the effective management of OM-NSCLC.

Keywords: Oligometastatic $(\mathrm{OM})$; lung cancer; surgery; prognosis; systematic review

Submitted Oct 16, 2020. Accepted for publication Apr 19, 2021.

doi: $10.21037 /$ tlcr-20-1123

View this article at: http://dx.doi.org/10.21037/tlcr-20-1123

^ ORCID: Luca Bertolaccini, 0000-0002-1153-3334; Lorenzo Spaggiari, 0000-0002-1068-3541. 


\section{Introduction}

In 2018, 470,000 new cases of non-small cell lung cancer (NSCLC) were diagnosed, and just about $50 \%$ of patients have already staged IV at the diagnosis for distant metastasis (DM) $(1,2)$. Stage IV NSCLC usually has a poor prognosis with a median survival of about $8-11$ months $(3,4)$ and is considered inoperable and often treated only with a palliative therapeutic approach. Surgery is used only in very particular cases and is more of a diagnostic tool than a therapeutic option.

To date, however, this group of patients is extraordinarily heterogeneous, and according to the $8^{\text {th }}$ TNM Edition, the difference in survival in stage IV NSCLC is significantly related to the site and number of metastases (5).

Patients with oligo or "few" metastases and their different survival, probably related to better biology and less aggressiveness of the tumour, was first described more than 20 years ago by Hellman and Weichselbaum (6). Nevertheless, the terms oligometastasis $(\mathrm{OM})$ is still unclear and often misunderstood. Several clinical scenarios for OM-NSCLC patients and their possible treatments have been described based on different sites (one or multiple organs), the number of metastasis ( $\leq 5 v s . \leq 3)$, and timing of appearance of the DM (synchronous vs. metachronous) (1-8).

Since the concept of "OM disease" was introduced in the oncological scenario of NSCLC, the possible reluctance of oncologists towards the surgical approach for these patients has been weakened, in particular in the last decade thanks to the recent advances in the new medical treatments such as immunotherapy or target therapies, which have led to a significant improvement in survival $(5,8,9)$.

Even if many questions about OM-NSCLC have no definite answer yet, these patients progressively became a new category of stage IV NSCLC. The multimodality approach, including surgery, may improve the prognosis.

Indeed, this systematic review of the literature aims to investigate the clinical prognostic factors in OM-NSCLC surgically treated with radical intent. We present the following article in accordance with the PRISMA reporting checklist (available at http://dx.doi.org/10.21037/tlcr-20-1123).

\section{Material and methods}

This systematic review is reported following the Preferred Reporting Items for Systematic Reviews and Meta-Analyses (PRISMA) statement (10). A search strategy using a combination of free-text words, relevant $\mathrm{MeSH}$ headings and appropriate restrictions (time limit: from January 1997 to March 2020, language: English) was designed. A systematic literature search was performed in EMBASE (via Ovid) [('non small cell lung cancer'/exp OR 'non small cell lung cancer' OR 'non small cell' OR 'nonsmall cell' OR nsclc) AND ('oligometastasis'/exp OR oligometastasis OR oligometasta* OR 'oligo-metastasis' OR 'oligo-metastases' OR 'oligo-metastatic' $O R$ oligoprogress* $O R$ 'oligo-progression' $O R$ 'oligo-progressive' $O R$ oligopersisten* $O R$ 'oligo-persistent' $O R$ 'oligopersistence' $O R$ oligorecurren* $O R$ 'oligo-recurrent' $O R$ 'oligo-recurrence' OR 'isolated metastasis' OR 'isolated metastases' OR 'limited metastasis' OR 'limited metastases' OR 'single organ metastasis' $O R$ 'single organ metastases' $O R$ 'solitary metastasis' $O R$ 'solitary metastases') AND ('prognosis'/exp OR prognosis OR prognoses OR 'prognostic factor'/exp OR 'prognostic factor' OR prognostic) OR (('prognosis'/exp OR prognosis OR prognoses OR 'prognostic factor'/exp OR 'prognostic factor' OR prognostic) AND ('oligometastatic non small cell lung cancer'/exp $O R$ 'oligometastatic non small cell lung cancer'))) AND [embase]/ lim NOT ([embase]/lim AND [medline]/lim)], MEDLINE (via PubMed) [(prognosis OR prognoses OR prognostic) AND (non small cell lung cancer OR "non small cell" OR "nonsmall cell" OR nsclc) AND (oligometasta* OR "oligo-metastasis" OR "oligo-metastases" OR "oligo-metastatic" OR oligoprogress* OR "oligo-progression" OR "oligo-progressive" OR oligopersisten* OR "oligo-persistent" OR "oligopersistence" OR oligorecurren* OR "oligo-recurrent" OR "oligo-recurrence" OR "isolated metastasis" OR "isolated metastases" OR "limited metastasis" OR "limited metastases" OR "single organ metastasis" OR "single organ metastases" OR "solitary metastasis" OR "solitary metastases")] and Cochrane CENTRAL [(oligometasta* OR "oligo-metastasis" OR "oligo-metastases" OR "oligo-metastatic" OR oligoprogress* OR "oligo-progression" OR "oligo-progressive" OR oligopersisten* OR "oligo-persistent" OR "oligopersistence" $O R$ oligorecurren* OR "oligo-recurrent" $O R$ "oligo-recurrence" OR "isolated metastasis" OR "isolated metastases" OR "limited metastasis" OR "limited metastases" OR "single organ metastasis" $O R$ "single organ metastases" OR "solitary metastasis" OR "solitary metastases") in Title Abstract Keyword AND ("nonsmall cell" OR "non small cell" OR NSCLC)]. Eligible studies were observational, describing clinical prognostic factors in OM-NSCLC wholly surgically treated with radical intent. Letters, editorials, case studies, expert opinions, metaanalyses, and reviews were all exempt from consideration. Our search strategy yielded documents, which were then imported into reference management software. In the event of a tie, the most recent paper was chosen. Based on the eligibility requirements, two authors independently 
evaluated each identified research. When data from different studies overlapped, the most insightful study was selected. With the assistance of a third investigator, disputes were addressed and settled by consensus. Potentially qualified papers were subjected to an in-depth full-text examination after preliminary title/abstract screening to identify studies for inclusion in the systematic review. Data extracted included: study characteristics, baseline patient characteristics, primary and secondary outcomes.

The Cochrane's Collaboration Tool was used to determine the risk of bias for included studies' primary outcome (11). The risk of bias due to incomplete outcome data was evaluated at an outcome level.

However, at the study stage, the possibility of bias due to sequence generation, allocation concealment, blinding, selective reporting, or funding was assessed. Two independent observers calculated the probability of bias, and differences were resolved through dialogue and consensus. Details of the systematic review protocol were entered into the International Prospective Registry of Systematic Reviews (PROSPERO) (12). We conducted a pooled analysis aimed at assessing the main discrepancies in the selected studies. A total of 517 patients who underwent surgery for OM lung cancer were included. The age ranged from 33 to 84 years, and the median was comparable. Demographics and baseline characteristics were adequately balanced in all studies. The main characteristics are presented in Table 1.

\section{Statistical analysis}

The Mantel-Haenszel formula was used to produce pooled impact estimates in the form of risk ratios (RR) and their $95 \%$ confidence intervals for dichotomous variables. The mean differences (MD) for continuous outcomes were pooled and weighted by generic inverse variance before being calculated using random-effects modelling. When a continuous outcome was reported as a median, range, or interquartile range in some studies, means and standard deviations were calculated using methods defined in the literature (22). The studies' methodological consistency in this analysis was evaluated using the Cochrane Collaboration risk of bias tool (11). Factors that were assessed included: random sequence generation, allocation concealment, blinding, incomplete outcome data, selective outcome reporting, and other potential sources of bias. Benefit evaluation was performed using Pareto optimal analysis $(23,24)$. Data analysis was performed using Review
Manager 5.3 (Nordic Cochrane Centre, Copenhagen, Denmark) (25) and RStudio (26).

\section{Results}

A total of 637 papers were found using the given search terms. Twelve papers were excluded due to duplication, leaving 625 articles. 8 disagreements were discussed and solved with the help of a third reviewer. The preliminary title/abstract screening restricted our search to 16 potentially eligible papers. According to our inclusion criteria, 7 articles were excluded at full-text reviewing, and 9 studies (13-21) were selected for the systematic review (Figure 1). A summary of the risk of bias for each included study is shown in Figure 2.

Median overall survival (OS) was 51.8 months and varied from 21.1 months (20) to 60 months (16), but results were not statistically significant. The disease-free survival (DFS) was reported only by two studies recently published $(14,18)$. The first reported a $21 \% 5$-year DFS and the second $23 \%$.

At the analysis, the positive prognostic factors for survival were smoking cessation, age $<60$, a histologic grade of G1/G2, pN0. The presence of extra-brain OM and multiple metastases negatively affected survival. For the formal presentation of our systematic review and the show of variations in score values provided by different target prediction tools in the recurrent method, we used the Pareto analysis. All the Pareto fronts produced provided by different target prediction tools were convex, as predicted from their representation as approximations of the correct Pareto fronts (Figure 3). The group had a favourable multicriteria analysis. . Nonetheless, the definition of an optimum solution for the OMTS-NSCLC cannot be described without additional constraints based on these assumptions.

\section{Discussion}

Since the introduction of target therapies and the significant increase in OM-NSCLC patients' survival, it becomes mandatory to find a common direction on stage IV patients' therapeutic approach for the OM cohort. However, the clinical heterogeneity and the multiple therapeutic strategies for these patients make it difficult to find a common thread. Besides, most of the literature studies are retrospective and based on insufficient patients, making it challenging to define guidelines for this type of patient (27).

In this systematic review, we tried to investigate the clinical prognostic factors in OM-NSCLC radically treated 


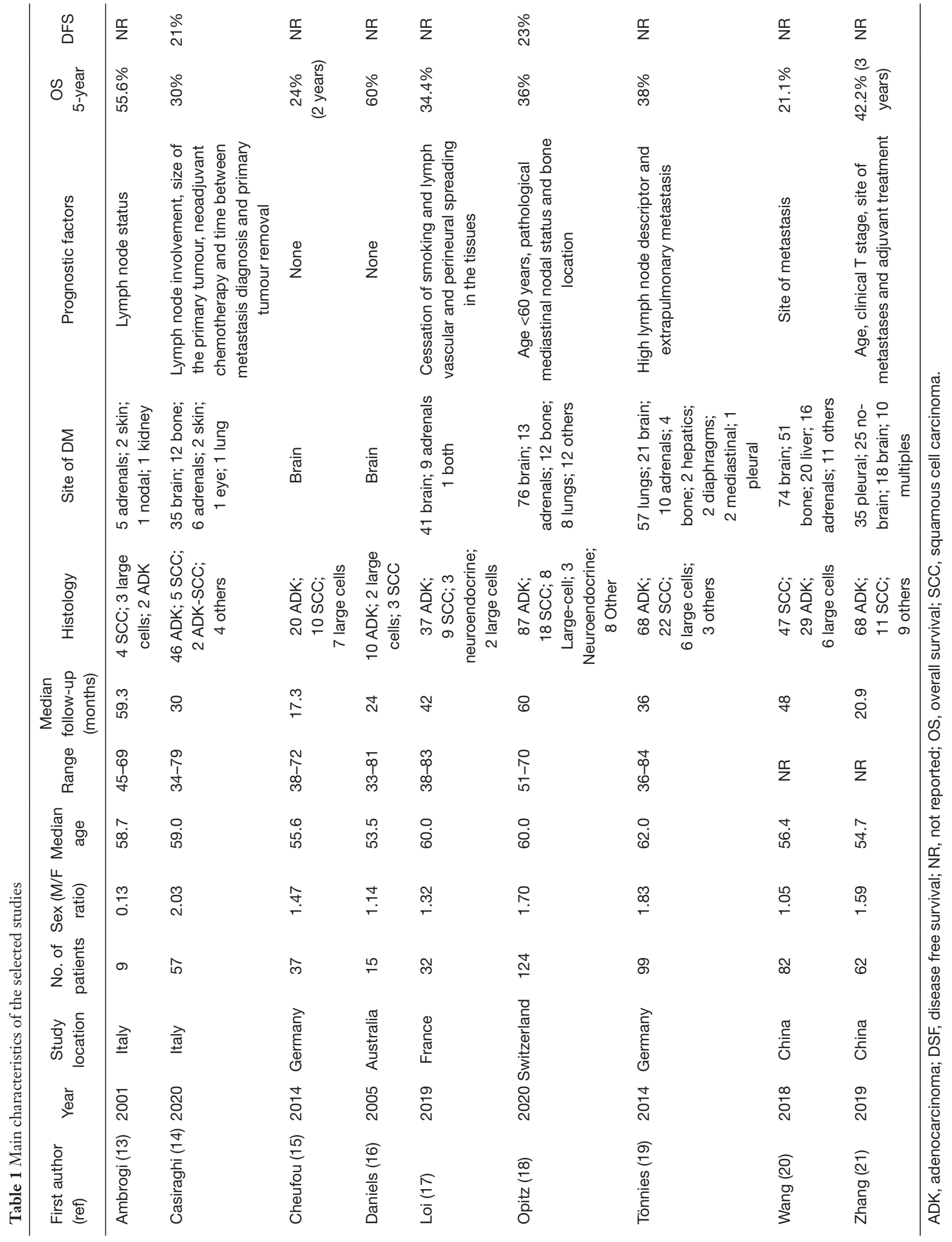




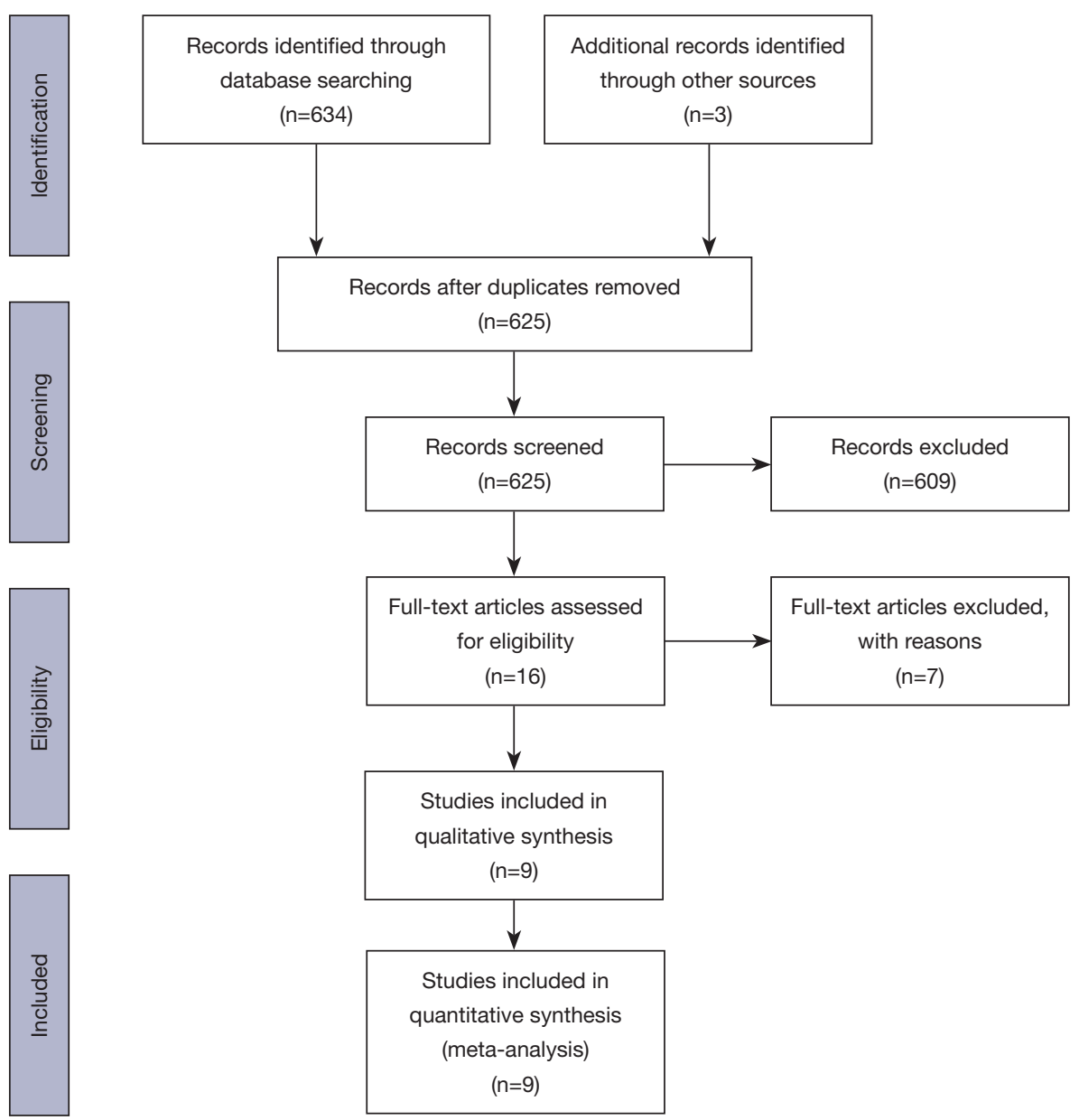

Figure 1 PRISMA flow chart.

to find a common denominator in such a heterogeneous group of patients for a better selection.

To date, the control of the primary disease and its distant metastases are the most important prognostic factors of OM-NSCLC. Many factors could influence the OS, even if only local treatments are associated with a significant improvement (28) in a multidisciplinary setting. Suppose we considered that a possible disease progression is usually more likely to occur at the DM sites at diagnosis rather than in new sites. In that case, patients with OM-NSCLC could benefit in terms of survival from the local treatment of the metastasis, such as surgery or radiotherapy (29-31).

According to our results, OM patients who were radically treated had a more robust OS and a significant prognostic difference depending on the metastatic site (i.e., a favourable factor in brain lesions) and the timing of presentation (synchronous vs. metachronous) (1,28). Besides, OM patients at diagnosis had a better OS than patients with oligorecurrence and/or oligoprogression during or after medical treatments (29). Then, positive prognostic factors for survival included single organ metastasis, the pN0 status, smoking cessation, age $<60$, and histologic grade of G1/G2. Indeed, a multidisciplinary strategy, including surgery, for patients with multiple sites of OM-NSCLC, should always be carefully considered on a case-by-case basis. When it comes to merging data from multiple studies, choosing between metaanalysis and pooled analysis is essential. The first approach is unquestionably less expensive and faster, but it suffers from performance limitations due to a lack of raw data. The basic criteria for performing a pooled study should be used when the exposure or outcome variables are difficult to measure and record, when there are several unpublished studies in the field, and when the monitoring of potential confounders is likely to be improved. The principle of Pareto efficiency (or 


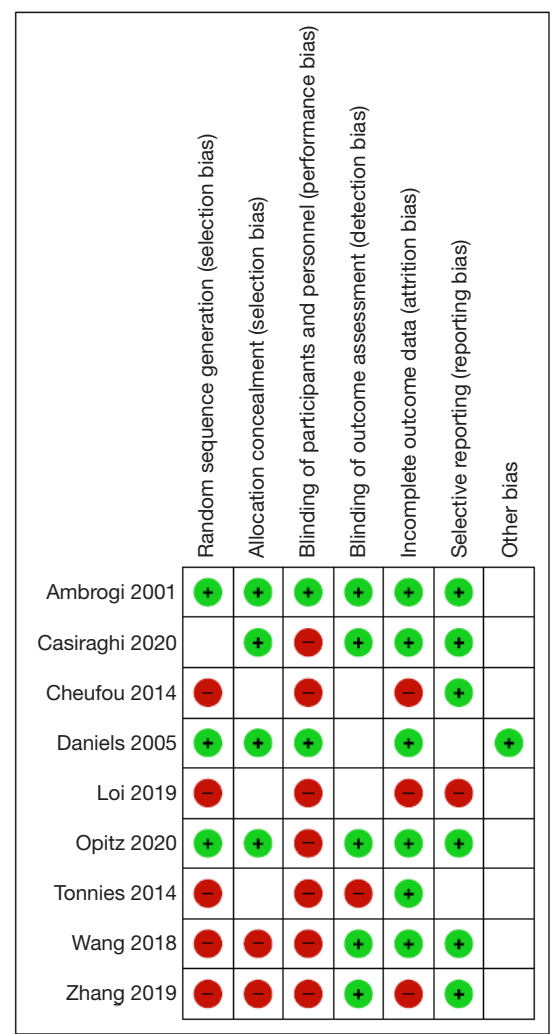

Figure 2 Risk of bias summary: Review the authors' evaluations of each risk of bias item for each study included.

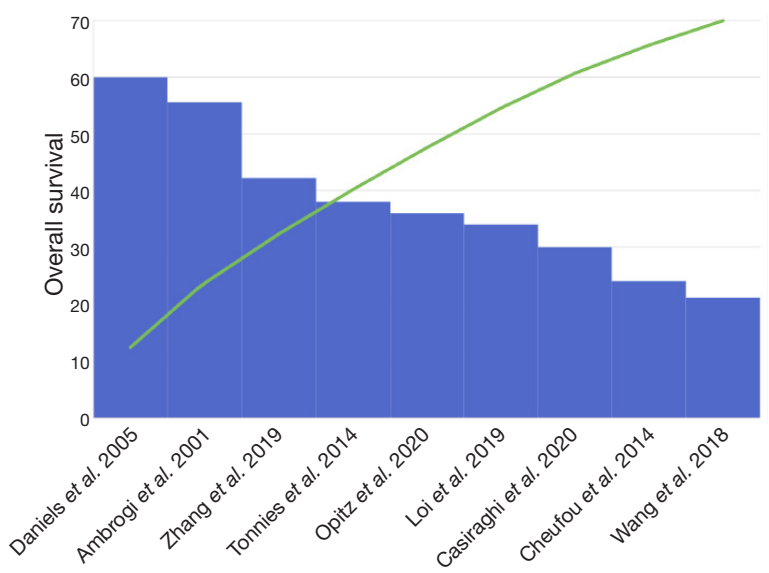

Figure 3 Pareto analysis of the pooled group.

Pareto optimality) is a tool for assessing the efficiency of a series of decisions taken by the participants in game theory and economics. Pareto diagrams may be used in medical process design to recognize mistakes, defects, and accidents, as well as in the implementation of a framework to minimize the risk of medical treatment and the review of performance data in health organizations.

Despite the absence of randomized prospective clinical trials evaluating the therapy sequence (metastases care before/after surgical operation and before/after medical treatment), brain metastases are generally treated before lung resection. On the other hand, adrenal gland metastases are treated after resection to maintain adrenocortical activity for lung surgery. Unfortunately, both the timing and the type of induction treatments, even if already demonstrated to be related to local control and clinical downstaging (32), could not be meta-analysed due to the population's heterogeneity included in the studies.

Another important key point is the biological feature of the tumour since, in the future, it might be able to distinguish the $\mathrm{OM}$ cohort from generally stage IV disease, as well as the differences between an indolent and an aggressive tumour. However, it also is the target of future therapy (in addition to local control) for patients who harbour sensitising driver mutations.

Our study presents some limitations: the studies reviewed were incredibly diverse, and some potentially essential studies were likely ignored. Given our best efforts, we could not find all acceptable facts due to our extensive searches lacking the so-called grey literature (dissertations, conference abstract, book chapters, and policy documents). The effect of grey literature, on the other hand, should not be considered. Second, the technique used is quite different. Because of the various disease states and operations involved, the mortality analyses focused on these studies (which involve all events) cannot be used to draw any conclusions.

In the absence of randomized data to help describe the effective treatment of OM-NSCLC, aggressive therapy should be used in otherwise stable patients with synchronous (or metachronous) extrathoracic M1 disease and no intrathoracic lymph node involvement.

\section{Acknowledgments}

The authors would like to thank Susan Jane West for editing the English text and William Russell-Edu (Librarian, IEO European Institute of Oncology IRCCS, Milan) to conduct the certified search of the systematic review.

Funding: This work was partially supported by the Italian Ministry of Health with Ricerca Corrente and 5×1,000 funds.

\section{Footnote}

Provenance and Peer Review: This article was commissioned 
by the Guest Editors (Maurizio Infante \& Thierry Berghmans) for the series "Oligometastatic NSCLC: definition and treatment opportunities" published in Translational Lung Cancer Research. The article has undergone external peer review.

Reporting Checklist: The authors have completed the PRISMA reporting checklist. Available at http://dx.doi. org/10.21037/tlcr-20-1123

Conflicts of Interest: All authors have completed the ICMJE uniform disclosure form (available at http://dx.doi. org/10.21037/tlcr-20-1123). The series "Oligometastatic NSCLC: definition and treatment opportunities" was commissioned by the editorial office without any funding or sponsorship. The authors have no other conflicts of interest to declare.

Ethical Statement: The authors are accountable for all aspects of the work in ensuring that questions related to the accuracy or integrity of any part of the work are appropriately investigated and resolved.

Open Access Statement: This is an Open Access article distributed in accordance with the Creative Commons Attribution-NonCommercial-NoDerivs 4.0 International License (CC BY-NC-ND 4.0), which permits the noncommercial replication and distribution of the article with the strict proviso that no changes or edits are made and the original work is properly cited (including links to both the formal publication through the relevant DOI and the license). See: https://creativecommons.org/licenses/by-nc-nd/4.0/.

\section{References}

1. Griffioen GHMJ, Toguri D, Dahele M, et al. Radical treatment of synchronous oligometastatic non-small cell lung carcinoma (NSCLC): patient outcomes and prognostic factors. Lung Cancer 2013;82:95-102.

2. Ferlay J, Colombet M, Soerjomataram I, et al. Cancer incidence and mortality patterns in Europe: Estimates for 40 countries and 25 major cancers in 2018. Eur J Cancer 2018;103:356-87.

3. Grossi F, Kubota K, Cappuzzo F, et al. Future Scenarios for the Treatment of Advanced Non-Small Cell Lung Cancer: Focus on Taxane-Containing Regimens. Oncologist 2010;15:1102-12.

4. American Cancer Society, Cancer Facts \& Figures 2014.
Cancer Facts Fig 2014. doi: 10.1177/0300985809357753.

5. Yang JCH, $\mathrm{Wu}$ YL, Schuler M, et al. Afatinib versus cisplatin-based chemotherapy for EGFR mutation-positive lung adenocarcinoma (LUX-Lung 3 and LUX-Lung 6): Analysis of overall survival data from two randomised, phase 3 trials. Lancet Oncol 2015;16:141-51.

6. Hellman S, Weichselbaum RR. Oligometastases. J Clin Oncol 1995;13:8-10.

7. Richard PJ, Rengan R. Oligometastatic non-small-cell lung cancer: current treatment strategies. Lung Cancer 2016;7:129-40.

8. Shaw AT, Yeap BY, Solomon BJ, et al. Effect of crizotinib on overall survival in patients with advanced non-small-cell lung cancer harbouring ALK gene rearrangement: A retrospective analysis. Lancet Oncol 2011;12:1004-12.

9. Paz-Ares LG, De Marinis F, Dediu M, et al. PARAMOUNT: Final overall survival results of the phase III study of maintenance pemetrexed versus placebo immediately after induction treatment with pemetrexed plus cisplatin for advanced nonsquamous non-small-cell lung cancer. J Clin Oncol 2013;31:2895-902.

10. Liberati A, Altman DG, Tetzlaff J, et al. The PRISMA statement for reporting systematic reviews and metaanalyses of studies that evaluate health care interventions: Explanation and elaboration. PLoS Med 2009;6:e1000100.

11. Higgins JPT, Altman DG, Gøtzsche PC, et al. The Cochrane Collaboration's tool for assessing risk of bias in randomised trials. BMJ 2011;343:d5928.

12. C. for R. and Dissemination, U. of York, PROSPERO, (n.d.). Available online: https://www.crd.york.ac.uk/ PROSPERO/ (accessed October 1, 2020).

13. Ambrogi V, Tonini G, Mineo TC. Prolonged survival after extracranial metastasectomy from synchronous resectable lung cancer. Ann Surg Oncol 2001;8:663-6.

14. Casiraghi M, Bertolaccini L, Sedda G, et al. Lung cancer surgery in oligometastatic patients: outcome and survival. Eur J Cardiothorac Surg 2020;57:1173-80.

15. Cheufou DH, Welter S, Chalvatzoulis E, et al. Surgery of primary lung cancer with oligometastatic M1b synchronous single brain metastasis: Analysis of 37 cases. Thorac Cardiovasc Surg 2014;62:612-5.

16. Daniels M, Wright GM. Complete resection of non-smallcell lung cancer and oligo-metastatic brain disease. ANZ J Surg 2005;75:963-6.

17. Loi M, Mazzella A, Mansuet-Lupo A, et al. Synchronous Oligometastatic Lung Cancer Deserves a Dedicated Management. Ann Thorac Surg 2019;107:1053-9. 
18. Opitz I, Patella M, Payrard L, et al. Prognostic factors of oligometastatic non-small-cell lung cancer following radical therapy: a multicentre analysis. Eur J Cardiothorac Surg 2020;57:1166-72.

19. Tönnies M, Pfannschmidt J, Bauer TT, et al. Metastasectomy for synchronous solitary non-small cell lung cancer metastases. Ann Thorac Surg 2014;98:249-56.

20. Wang Z, Gao SG, Xue Q, et al. Surgery of primary nonsmall cell lung cancer with oligometastasis: Analysis of 172 cases. J Thorac Dis 2018;10:6540-6.

21. Zhang C, Wang L, Li W, et al. Surgical outcomes of stage IV non-small cell lung cancer: A single-center experience. J Thorac Dis 2019;11:5463-73.

22. Guyot P, Ades AE, Ouwens MJNM, et al. Enhanced secondary analysis of survival data: Reconstructing the data from published Kaplan-Meier survival curves. BMC Med Res Methodol 2012;12:9.

23. Ottosson RO, Engström PE, Sjöström D, et al. The feasibility of using Pareto fronts for comparison of treatment planning systems and delivery techniques. Acta Oncol 2009; 48:233-7.

24. Wright A, Bates DW. Distribution of problems medications and lab results in electronic health records: The pareto principle at work. Appl Clin Inform 2010;1:32-37.

25. The Cochrane Collaboration, Review Manager (RevMan). Version 5.3. Copenhagen: The Nordic Cochrane Centre, (2014). Available online: https://training.cochrane.org/ online-learning/core-software-cochrane-reviews/revman (accessed October 1, 2020).

26. R studio, (n.d.). Available online: https://rstudio.com/ (accessed October 1, 2020).

27. Bertolaccini L, Spaggiari L. The radical approach to the oligometastatic not small cell lung cancer patient: The radical approach to the oligometastatic not small cell lung cancer patient: which? how? when? where? J Thorac Dis 2019;11:S2023-S2025.

28. Ashworth A, Rodrigues G, Boldt G, et al. Is there an oligometastatic state in non-small cell lung cancer? A systematic review of the literature. Lung Cancer 2013;82:197-203.

29. Berzenji L, Van Schil PE. Oligometastatic non-small cell lung cancer: is there a role for locoregional therapy? J Thorac Dis 2017;9:1814-7.

30. Gomez DR, Blumenschein GR, Lee JJ, et al. Local consolidative therapy versus maintenance therapy or observation for patients with oligometastatic non-smallcell lung cancer without progression after first-line systemic therapy: a multicentre, randomised, controlled, phase 2 study. Lancet Oncol 2016;17:1672-82.

31. Folkert MR, Timmerman R, Berzenji L, et al. Surgical approach in the oligometastatic patient. Lung Cancer 2016;82:186-93.

32. Congedo MT, Cesario A, Lococo F, et al. Surgery for oligometastatic non-small cell lung cancer: Longterm results from a single center experience. J Thorac Cardiovasc Surg 2012;144:444-52.
Cite this article as: Bertolaccini L, Casiraghi M, Sedda G, de Marinis F, Spaggiari L. Clinical prognostic factors in surgically treated oligometastatic non-small cell lung cancer: a systematic review. Transl Lung Cancer Res 2021;10(7):34013408. doi: 10.21037/tlcr-20-1123 